\title{
EMPLEO DEL MODELO SRNS PARA PREDECIR LA GANANCIA DE PESO EN OVINOS MACHOS PELIBUEY EN CRECIMIENTO
}

\section{SRNS MODEL ADEQUACY FOR BODY WEIGHT GAIN PREDICTING IN GROWING PELIBUEYLAMBMALES}

\author{
Duarte-Vera, F. ${ }^{* 1,2}$, C. Sandoval-Castro ${ }^{2 A}$ y L. Sarmiento-Franco ${ }^{2 B}$ \\ ${ }^{1}$ Campo Experimental Mocochá. Centro de Investigación Regional del Sureste. INIFAP. Km 15 Carr. Mérida- \\ Baca. Yucatán. México. *fduarte53@yahoo.com.mx \\ ${ }^{2}$ Facultad de Medicina Veterinaria y Zootecnia. Universidad Autónoma de Yucatán. Mérida. Yucatán. \\ México. ${ }^{2 A}$ ccastro@uady.mx; ${ }^{2 B}$ fsarmien@uady.mx
}

\section{Palabras clave adicionales}

Modelos de predicción. Metabolismo basal. Índice de madurez.

\section{RESUMEN}

El objetivo del trabajo fue evaluar los ajustes en el índice de madurez, el valor para metabolismo basal y el peso vivo vacío, sobre la capacidad del modelo Small Ruminant Nutrition System (SRNS) para predecir la ganancia diaria de peso vivo (GDP) en ovinos Pelibuey durante la etapa de crecimiento. El trabajo se realizó en la Facultad de Medicina Veterinaria y Zootecnia de la Universidad Autónoma de Yucatán, México. Se utilizó una base de datos constituida por 26 experimentos y 77 dietas, con un total de 1112 ovinos Pelibuey machos. Para el índice de madurez $(P)$, el peso maduro se estimó mediante una curva de crecimiento, siendo éste de $66 \mathrm{~kg}$. Para la evaluación del modelo, se utilizó el programa Model Evaluation System con los siguientes indicadores: El análisis de la regresión lineal; el error estándar de la estimación (MSE); el coeficiente de concordancia de la correlación (CCC); la media mínimo cuadrática del error de la predicción, (MSEP); la eficiencia de modelo (MEF), y el coeficiente de determinación del modelo (CD). El modelo sin ajustar, subestima la predicción de la ganancia de peso vivo. El índice de madurez ajustado con el peso maduro de machos Pelibuey y el valor para el metabolismo basal de 0,039 Mcal de $\mathrm{EM} / \mathrm{kg}^{0,75}$ mejoraron la precisión y exactitud del modelo sobre la predicción de la GDP en machos Pelibuey. Se concluye que el valor del metabolismo basal es menor en ovinos Pelibuey que en razas lanares y que el valor ajustado del metabolismo basal en la ecua-

Recibido: 16-1-08. Aceptado: 17-9-08.

\section{ADDITIONAL KEYWORDS}

Prediction models. Basal metabolism. Maturity index.

ción para estimar las necesidades de mantenimiento en el SRNS mejora la predicción de la GDP en machos Pelibuey en crecimiento y que el ajuste por el contenido gastrointestinal no presenta ventajas para utilizarse en el modelo.

\section{SUMMARY}

The objective of the present study was to evaluate adjustments in the maturity index, the value for basal metabolism and the empty live weight proposed for the Small Ruminant Nutrition System (SRNS) model on its capacity to predict the daily weight gain (DWG) of growing Pelibuey rams. The work was carried out at the Faculty of Veterinary Medicine and Animal Science of the University of Yucatan, Mexico. A data base composed by 26 experiments, 77 diets, with a total of 1112 male Pelibuey lambs was used. In order to calculate the index of body maturity, the mature weight was taken as $66 \mathrm{~kg}$. The Model Evaluation System software was used to evaluate the model by means of: Linear regression analysis; the standard error of the estimation (MSE); the coefficient of agreement of the correlation (CCC); the mean standard error of the prediction, (MSEP); the model efficiency (MEF), and model coefficient of determination (CD). The model without adjustments underestimated DWG. Adjustment for maturity (mature weight) and a basal metabolism of $0.039 \mathrm{Mcal} \mathrm{ME} / \mathrm{kg}^{0.75}$, improved the model's 


\section{DUARTE-VERA, SANDOVAL-CASTROYSARMIENTO-FRANCO}

precision and accuracy for DWG prediction of growing Pelibuey males. It was concluded that basal metabolism in Pelibuey sheep is lower than in wool breeds. Using the adjusted basal metabolism value in the equation to estimate maintenance requirements improved the SRNS DWG prediction and the adjustments for estimated gastrointestinal content did not improved model predictions.

\section{INTRODUCCIÓN}

En México la producción ovina es una de las principales actividades del sector agropecuario. El auge de esta actividad en las regiones tropicales se ve reflejado, por un lado en el incremento del inventario de animales y por otro, en una mayor producción debido a la aplicación de programas de manejo reproductivo, nutricional, sanitario y de mejoramiento genético, principalmente de la raza Pelibuey. La intensificación de los sistemas de producción, conlleva la generación de tecnología que permita la expresión del potencial genético de los animales con una buena eficiencia en la utilización de los recursos alimenticios; para ello, es indispensable actualizar los requerimientos nutricionales de esta raza en sus diferentes etapas productivas. Se han desarrollado numerosos modelos para predecir los requerimientos nutricionales de los ovinos, sin embargo éstos han empleado para ello razas lanares e información de forrajes y alimentos de clima templado (NRC, 1985; ARC, 1980; AFRC, 1993; CSIRO, 1990; INRA, 1978). Cannas et al. (2004) desarrollaron un modelo mecanístico basado en el modelo de carbohidratos y proteínas netas de la Universidad de Cornell (Cornell Net Carbohydrate and Protein System; CNCPS), modificándolo para usarse en ovinos de lana (CNCPS-S), prediciendo las necesidades energéticas con énfasis en lactación. Este modelo, sirvió de base para desarrollar el modelo Small Ruminant Nutrition System (SRNS), el cual predice los requerimientos de energía, proteína, calcio y fósforo, de acuerdo a las características del animal y del medio. Los valores biológicos del alimento, son predichos tomando en cuenta las fracciones de los carbohidratos y de la proteína, su tasa de pasaje y fermentación ruminal, el crecimiento microbial y el aporte de fibra físicamente efectiva (Tedeschi, 2007). La aplicación de este modelo en áreas tropicales con ovinos de pelo como la raza Pelibuey, sugiere la conveniencia de realizar algunos ajustes para mejorar la predicción de la ganancia de peso vivo (Duarte-Vera et al., 2008).

El objetivo del presente trabajo fue evaluar algunas adecuaciones propuestas para el modelo SRNS (índice de madurez, ajuste del valor energético para metabolismo basal y del peso vivo vacío), sobre la capacidad de predicción de la ganancia de peso vivo de ovinos Pelibuey durante la etapa de crecimiento.

\section{MATERIAL Y MÉTODOS}

El trabajo se realizó en la Facultad de Medicina Veterinaria y Zootecnia de la Universidad Autónoma de Yucatán, ubicada en Xmatkuil, municipio de Mérida, Yucatán, México.

Se utilizó una base de datos de un trabajo anterior (Duarte-Vera et al., 2008), constituida por 26 experimentos y 77 dietas, con un total de 1112 ovinos Pelibuey machos, en la etapa de crecimiento-finalización. Los experimentos fueron diseñados para evaluar la inclusión de subproductos agroindustriales como melaza y pastas de oleaginosas en las dietas, así como la de desechos orgánicos (excretas de pollos de engorda y cerdaza), cultivos alternativos como la yuca y el ensilaje de maíz, de sorgo o de yuca sobre la ganancia de peso vivo, el consumo de alimento y la conversión alimenticia. El diseño experimental más empleado fue, el completamente al azar, eventualmente con arreglo factorial (niveles de proteína y energía); el número de repeticiones fluctuó entre tres y seis por tratamiento, siendo la unidad experimental el animal, en la mayoría de los casos, o el corral donde se alojaban los 
borregos (dos o tres). Las principales características de los animales se presentan en la tabla I. Los datos sobre la composición de alimentos, se obtuvieron de la biblioteca tropical del CNCPS (Tedeschi et al., 2002) y de tablas de composición de alimentos del NRC (1996) para valores del total de nutrientes digestibles (TND), fibra detergente neutro (FDN) y fibra detergente neutro efectiva (FDNe), de los ingredientes que no se encontraron en la primera fuente. Cuando alguno de los ingredientes no se encontró en ninguna de las fuentes mencionadas, para obtener los valores específicos, como el porcentaje de fibra detergente neutro efectiva, el porcentaje de proteína indigestible u otros, se tomaron valores de otros ingredientes del mismo tipo y cuya composición química era similar en la biblioteca tropical del CNCPS (Tedeschi et al., 2002) con la finalidad de poder calcular el aporte total de la dieta y utilizar los datos para aplicar el modelo. Cuando esto no fue posible, no se consideró la dieta para el análisis correspondiente en la base de datos.

En los trabajos revisados, donde no se indicó la composición nutricional de la dieta integral, sino únicamente la del suplemento ofrecido como parte de la ración (Bores et al., 1988; Partida, 1983; Romano et al., 1983), se volvieron a calcular las dietas, incluyendo al forraje, para estimar el porcentaje de proteína cruda y la energía metabolizable contenida en la materia seca del total de la ración; posteriormente se estimó el consumo de energía por animal por día en Mcal de EM, considerando el contenido de energía de la materia seca de la ración total y el alimento diario consumido por animal .

El peso vivo se estimó como el promedio entre el peso de los animales al inicio de los experimentos y el peso al final de los mismos; este dato sirvió para calcular el peso reducido y el peso metabólico en cada una de las pruebas. Para calcular el índice de madurez $(\mathrm{P})$, el peso maduro se estimó mediante una curva de crecimiento para machos Pelibuey (Duarte et al., 2007).
Tabla I. Características generales de los animales de la base de datos, $(n=1112)$. (General features of animal data base $(n=1112)$ ).

\begin{tabular}{lcccccc}
\hline & PI & PF & PP & PV & PM & E \\
\hline Media & 21,6 & 33,0 & 27,3 & 26,2 & 11,6 & 0,5 \\
Máximo & 34,0 & 47,6 & 38,0 & 36,4 & 14,8 & 2 \\
Mínimo & 12,0 & 12,5 & 12,5 & 12 & 6,4 & 0,3 \\
\hline
\end{tabular}

PI: Peso inicial (kg); PF: Peso final (kg); PP: Peso promedio (kg); PV: Peso vacío (kg); PM: Peso metabólico ( $\left.\mathrm{kg}^{0,75}\right)$; E: Edad (años).

Para obtener los datos ambientales, se consultó la información meteorológica de las estaciones más cercanas al lugar donde se llevó a cabo el experimento y se incluyó la temperatura mensual promedio del mes anterior al inicio del trabajo; cuando esto no fue posible, se consideró la temperatura promedio de los últimos 40 años del Observatorio Meteorológico de la ciudad de Mérida Yucatán, México(CONAGUA, 2005),

La información se analizó empleando el modelo SRNS, evaluando el efecto de los ajustes propuestos tomando como indicador la capacidad de predicción de la ganancia de peso vivo en ovinos Pelibuey machos, quedando los siguientes modelos:

\section{ModeloSRNS}

Para calcular la ganancia de peso vivo (ADG), se empleó la ecuación propuesta por Cannas et al. (2004):

$A D G=R E / E V G(0,92)$

En donde:

$\mathrm{ADG}=$ ganancia diaria promedio ( $\mathrm{kg} / \mathrm{día})$,

$\mathrm{RE}=$ energía neta disponible para ganancia (Mcal/ día),

$E V G=$ contenido de energía en la ganancia de peso vacío $(0,851 \mathrm{SBW}, \mathrm{kg})$.

El modelo de Cannas et al. (2004) se basa en la ecuación propuesta por CSIRO (1990), modificada por Freer y Donnelly (1997) y es la siguiente: 


\section{DUARTE-VERA, SANDOVAL-CASTROYSARMIENTO-FRANCO}

$$
E V G=\left(6,7+2(L-1)+\frac{\underline{Z}_{1}-2(L-1)}{1+e^{-6(P-0,4)}}\right) 0,239
$$

Donde:

$\mathrm{L}=$ nivel de alimentación en múltiplos de $\mathrm{ME}_{\mathrm{m}}$ menos una unidad (Mcal/Mcal),

$Z_{1}=16,5$ (valor de ajuste para razas de borregos más magras en relación a la raza Merino),

$\mathrm{P}=$ Índice de madurez.

Para calcular la energía para el mantenimiento, se utilizó la ecuación de Cannas et al. (2004):

$E M_{m}=\left\{\left[S^{0} W^{0,75}\right.\right.$ a1 a2 $+\exp (-0,03$ AGE) $]+$ $\left.\left(-0,09 \mathrm{MEI} \mathrm{k}_{\mathrm{m}}\right)+\mathrm{ACT}+\mathrm{NE}_{\mathrm{mes}}+\mathrm{UREA}\right\} / \mathrm{k}_{\mathrm{m}}$

Donde:

$E M_{m}=$ requerimiento de energía metabolizable para el mantenimiento en Mcal/día,

$\mathrm{SBW}^{0,75}=$ peso metabólico reducido, equivalente al 96\% del PV ${ }^{0,75}$ lleno en kg,

a1= requerimiento de energía para el metabolismo basal (Mcal/kg PV ${ }^{0,75}$ ),

$\mathrm{a} 2=$ factor de ajuste por efecto de temperatura ambiental,

$\exp (-0,03$ AGE $)=$ expresión exponencial para ajuste de edad en años,

MEI= consumo de EM en Mcal por día,

$\mathrm{k}_{\mathrm{m}}=$ coeficiente de eficiencia en la utilización de la energía para mantenimiento,

$\mathrm{ACT}=$ efecto de la actividad sobre los requerimientos para mantenimiento en Mcal/día (considerando que los animales caminaron $50 \mathrm{~m}$ diarios en terreno plano),

$\mathrm{NE}_{\text {mes }}=$ Estimación de energía adicional para equilibrar el estrés por frío,

UREA = costo energético en Mcal EM $M_{m}$ para eliminar el excedente de $\mathrm{N}$ como urea.

Para la predicción de la eficiencia del uso de la energía para ganancia de peso $\left(\mathrm{k}_{\mathrm{g}}\right)$, se utilizó la ecuación propuesta por Cannas et al. (2006), y que a continuación se presenta:

$$
K_{g}=\frac{3}{4+11(R E p)}
$$

Donde:

$\mathrm{RE} p=$ proporción de la energía de la proteína en la energía neta para ganancia (Mcal/Mcal).
Cabe mencionar que no se consideró el efecto de raza y sexo como en el modelo CNCPS para bovinos, debido a que estudios previos sostienen que la variación en los requerimientos de mantenimiento dependen del plano de nutrición dentro de animales de una misma raza, siendo mayor el requerimiento de energía para el mantenimiento en ovinos alimentados ad libitum que cuando la alimentación es restringida (Olthoff et al., 1989). Adicionalmente, no se consideró necesario incluir el factor de ajuste para el efecto de estrés por frío $\left(\mathrm{NE}_{\text {mes }}\right)$ debido a las diferencias climáticas existentes entre el lugar de origen y las regiones tropicales, asumiendo que en estas últimas no hay estrés por frío.

\section{MODELOSRNS, AJUSTANDO EL FACTOR PARAMETABOLISMOBASAL}

Debido a que las variables de GDP y consumo de nutrimentos eran conocidos en cada una de las observaciones, se estimó el factor para el metabolismo basal (a1) de la ecuación para determinar las necesidades de energía para el mantenimiento (ecuación [3]), como sigue:

$$
\mathrm{a} 1=\left[\left(\mathrm{K}_{\mathrm{m}} \times \mathrm{ME}_{\mathrm{m}}\right)-(0,09 \times \mathrm{MEI} \times 0,64)-\mathrm{ACT}-\right.
$$

UREA]/SWB ${ }^{0,75} \times$ a2 $\times \exp (-0,03 \times$ AGE) [5]

El valor promedio obtenido mediante la aplicación de la ecuación anterior de 0,039 ( $\mathrm{n}=77$ dietas), se incluyó en la ecuación [1] y se comparó con los valores de 0,052 empleado por Cannas et al. (2006) y 0,062 Mcal $\mathrm{EM} / \mathrm{kg}^{0,75}$ (Cannas et al. (2004) con la finalidad de evaluar el ajuste sobre el metabolismo basal sobre la precisión y exactitud para predecir la ganancia de peso vivo en borregos Pelibuey en crecimiento.

\section{Modelo SRNS AJUSTANDO EL METABO-} LISMO BASAL Y ELPESO VIVO VACÍO(PVV):

Debido a que la cantidad de tejido corporal se relaciona con el peso vivo, y éste a su vez con el llenado del tracto gastrointestinal, que en ovinos Pelibuey es mayor

Archivos de zootecnia vol. 58, núm. 224, p. 674. 
que en las razas lanares (Martínez et al., 1987), con un promedio de $18 \%$ del peso vivo en los últimos (tabla I), se generó una relación lineal (empírica) entre el peso vivo vacío y el porcentaje de consumo de materia seca en relación al peso vivo, tomando valores relativos de peso vivo vacío mínimo de 80 y máximo de $96 \%$ y de 3 a $7 \%$ del consumo de materia seca en relación al peso vivo:

$P V V=P V(0,96)$

Si CMS $<3 \%$ PV

$P V V=P V(1,08-0,04 \times C M S)$ Si CMS $>3 \% P V$

Esta ecuación se incluyó en el modelo para evaluar el efecto de la variable peso vivo vacío, realizando las corridas con los tres valores de a1, adicionando el ajuste del PVV.

Para la evaluación de los modelos, se utilizaron varios indicadores de acuerdo con la metodología sugerida por Tedeschi (2006), empleando el Model Evaluation System (versión. 2.0.11,2006) : 1).-El análisis de la regresión lineal en donde se consideraron los valores observados en el eje de las $y$, debido a que estos contienen la variación natural debido a que los valores que predice el modelo son determinísticos y no tienen una variación al azar (Harrison, 1990; Mayer y Butler, 1993; Mayer et al., 1994). Se comprobó la linealidad de los datos, mediante un análisis de regresión lineal múltiple, para verificar la significancia de los efectos cuadráticos y cúbicos, utilizando el paquete estadístico SAS (1988).2).- El error estándar de la estimación (EEE), se utilizó como indicador para validar el modelo,(Analla, 1980). 3).- El coeficiente de concordancia de la correlación (CCC), apropiado para variables continuas (Cohen, 1960), que evalúa la exactitud $\left(C_{b}\right)$ y la precisión $\left({ }^{\wedge} P_{c}\right)$ simultáneamente (Tedeschi, 2006).4).La media mínimo cuadrática del error de la predicción, (MEP), junto con el EEE, miden la exactitud del modelo. 5).- La eficiencia del modelo (EM), y el coeficiente de determinación del modelo (CD), son indicadores que evalúan la idoneidad del modelo empleado (Tedeschi, 2006).

Los datos se sometieron a un análisis agrupando los datos de cada modelo, empleando el programa Model Evaluation System (2006; MES versión. 2.0.11); analizando por separado los efectos de incluir el costo energético para eliminar el excedente de $\mathrm{N}$ y el efecto del PVV en cada uno de los modelos ajustados con los tres valores de a1.

\section{RESULTADOS Y DISCUSIÓN}

Los valores para ganancia de peso vivo en la base de datos, tienen un rango muy amplio por el diseño mismo de los experimentos, habiendo casos en los que el aporte de nutrimentos limitó el crecimiento de los animales e incluso ocasionó la pérdida de peso. En contraste, en otros trabajos se observaron ganancias de peso vivo superiores a los $300 \mathrm{~g}$ por día.

El coeficiente de correlación entre la ganancia de peso observada y la calculada, fue altamente significativo $(\mathrm{p}<0,01)$, sin embargo, un alto coeficiente de correlación, no indica que la línea estimada de regresión sea un indicador adecuado del modelo, sobre todo si la relación no es lineal (Tedeschi, 2006); situación que no ocurrió en este caso, debido a que el componente parcial de la $\mathrm{r}^{2}$ fue prácticamente nulo con un valor de 0,00001 , por lo que la respuesta fue lineal, con un valor de la $\mathrm{r}^{2}$ total de 0,495 .

El consumo de materia seca observado fue ligeramente superior a lo que se indica en la literatura, así por ejemplo, para borregos de $30 \mathrm{~kg}$ con una ganancia de peso vivo de 295 g, el NRC (1985) estima un consumo de materia seca de $1,3 \mathrm{~kg}$, equivalente al $4,3 \%$ del peso vivo, mientras que el promedio de lo observado equivale al $4,6 \%$; esta diferencia se vio incrementada a medida que los animales aumentaron de peso, llegando a consumir el 5,3\% de materia seca respecto al peso vivo, al final de los experimentos (median=29).

El SRNS, predice la digestibilidad cuando el $\mathrm{pH}$ ruminal es de 6,2 o mayor y, para las 


\section{DUARTE-VERA, SANDOVAL-CASTROYSARMIENTO-FRANCO}

dietas con alto contenido de granos, considera la posibilidad de incluir en el modelo el efecto de ionóforos o moduladores de la fermentación ruminal, sin embargo esta información no se tiene en la mayoría de los experimentos de la base de datos por lo cual no se incluyó. Es importante señalar que en el CNCPS-S la predicción de la digestibilidad de la dieta está fuertemente influenciada por el pH ruminal (Cannas et al., 2004), y dado que la mayoría de las dietas de la base de datos tienen niveles altos de grano, es posible que el $\mathrm{pH}$ ruminal en los animales haya estado por debajo de 6,2 , y por lo tanto afectar negativamente la digestibilidad; esto daría como consecuencia una disminución en la disponibilidad de energía para el animaly, consecuentemente, predecir menores ganancias de peso vivo. Por otro lado, el balance de nitrógeno en el rumen fue positivo en general, con una media de 8,1 (tabla II); solamente 4 de las 77 dietas tuvieron resultados negativos que correspondieron a dietas con niveles bajos de proteína cruda (de 5 a 9\%); sin embargo, la diferencia en la predicción de la GDP en estos casos, respecto a la observada, es incluso menor a la desviación estándar de la media y se consi- dera que no afecta a los resultados globales.

La inclusión de peso maduro ajustado para ovinos Pelibuey en el modelo para calcular el índice de madurez, mejora la predicción de la GDP considerablemente. La actualización de este índice es una aportación importante ya que se puede emplear en otros modelos de predicción como el NRC (1985), en donde se utiliza para calcular los requerimientos de energía neta para ganancia de peso $\left(\mathrm{EN}_{\mathrm{g}}\right)$, mediante la siguiente ecuación:

$$
\mathrm{NE}_{\mathrm{g}}=276 \mathrm{LWG} \times \mathrm{W}^{0,75}
$$

donde:

LWG es la ganancia de peso vivo esperada y, $W^{0,75}$ es el peso maduro, considerado este de 115 $\mathrm{kg}$ e incrementando $21 \mathrm{kcal}$ por cada $10 \mathrm{~kg}$ de peso maduro menor.

Si consideramos que el peso maduro de un pelibuey es de $66 \mathrm{~kg}$ (Duarte et al., 2007), la energía retenida por los tejidos se incrementaría en $105 \mathrm{kcal}$ ya que la diferencia en el peso maduro es de $49 \mathrm{~kg}$, por lo cual el valor sería de 375 en vez de 276 en la fórmula anterior; esto podría explicar la

Tabla II. Valores observados en la base de datos y predichos con el modelo SRNS en el comportamiento de ovinos Pelibuey y principales características de las dietas $(n=77)$. (Observed and predicted values with SRNS model on Pelibuey performance and main diet features $(n=$ 77)).

\begin{tabular}{lccccccccc}
\hline & GDP observada & GDP esperada* & CMS & CEM & FDNe & PC & BNR* & Forraje & TND* \\
\hline Media & 171,6 & 122,5 & 1,3 & 3,2 & 13,83 & 13,5 & 8,10 & 34,1 & 51,1 \\
Máximo & 319,0 & 309,0 & 2,0 & 5,6 & 32,91 & 20,0 & 16,85 & 73,4 & 79,5 \\
Mínimo & $-46,0$ & $-14,0$ & 0,6 & 1,6 & 0,13 & 5,1 & $-3,67$ & 6,0 & 29,9 \\
DE & 72,0 & 62 & 0,3 & 0,83 & 6,90 & 2,8 & 5,70 & 17,4 & 10,6
\end{tabular}

GDP= ganancia diaria de peso $(\mathrm{g}) ; \mathrm{CMS}=$ consumo de materia seca (kg/animal/día); CEM= consumo de energía metabolizable (Mcal de EM/animal/día); FDNe= fibra detergente neutro efectiva como porcentaje de la FDN en la ración; $\mathrm{PC}=$ proteína cruda, como porcentaje de la materia seca; $\mathrm{BNR}=$ balance de nitrógeno en el rumen; Forraje= contenido de forraje como porcentaje de la materia seca de la dieta; TND= total de nutrimentos digestibles, como porcentaje de la materia seca; DE= desviación estándar de la media.

*Valores calculados por el SRNS.

Archivos de zootecnia vol. 58, núm. 224, p. 676. 
mejora en la predicción de la GDP por el modelo.

\section{MOdELOSRNS}

Los indicadores para la evaluación del modelo, se presentan en las tablas III y IV. La predicción de la ganancia de peso vivo está subestimada por el modelo (29\%), con relación a los datos observados. No obstante, hay que considerar que existen varios factores que pueden contribuir a ello, por ejemplo: el cálculo del consumo de energía por animal y día, se hace considerando el consumo total de materia seca y su contenido energético, sin embargo éste valor se estima, en la mayoría de los casos, mediante la composición nutricional de los ingredientes en las tablas disponibles y no mediante los análisis bromatológicos correspondientes. Dado que la energía para crecimiento $=$ EM consumida-EM de mantenimiento, otra posibilidad sería que los requerimientos de energía para el mantenimiento estuvieran sobreestimados, lo cual implica que al hacer los cálculos con el modelo, la energía disponible para el crecimiento sea menor y por lo tanto, la predicción hecha en cuanto a la ganancia de peso esté subestimada. Es posible que las diferencias raciales en cuanto a la conformación de los animales (peso maduro, porcentaje de grasa y hueso en la canal) también influyan sobre los resultados.

\section{MODELO SRNS AJUSTANDO EL FACTOR PARA METABOLISMOBASAL}

Si se considera que de la energía consumida y metabolizada, una buena parte se utiliza para el mantenimiento del animal (54\% en el caso de la base de datos analizada), y que uno de los principales factores que intervienen para calcular estas necesidades es el metabolismo basal, es importante actualizar los cálculos que permitan estimar mejor este factor. Cannas et al. (2006), proponen ajustarlo, disminuyendo el valor de 0,062 a 0,052 Mcal de EM/ $\mathrm{kg}^{0,075}$, para mejorar la predicción de la ganancia de peso vivo en ovinos de pelo con el CNCPS-S.

El valor promedio obtenido en el presente trabajo, al evaluar la ecuación [6] en cada una de las dietas empleadas en los experimentos $(n=77)$, fue de 0,039 Mcal de EM/ $\mathrm{kg}^{0,75}$, valor inferior al de 0,062 que considera el modelo original del CNCPS-S (Cannas et al., 2004), y al 0,052 que sugieren Cannas et al., 2006). Al emplear el valor de 0,039, mejoró la predicción de la ganancia de peso vivo en borregos Pelibuey con el modelo SRNS, apoyando la hipótesis de que las necesidades de energía para el metabolismo basal en esta raza, puede ser menor a las de razas lanares; como consecuencia, los requerimientos de energía para el mantenimiento de los borregos Pelibuey serían menores a los previamente reportados por otros investigadores (Bores et al., 2001; Solís et al., 1991). Esto podría deberse en parte, al mayor contenido del aparato gastrointestinal del ovino Pelibuey, comparado con el de razas lanares, aunado a un porcentaje mayor de hueso en relación al peso vivo del animal (30,5\% en Pelibuey y de 16 a 24\% en razas lanares; Martínez et al., 1985), dando como resultado una menor cantidad de tejido metabólicamente activo y por lo tanto, disminuyendo las necesidades de energía para el metabolismo basal. Así, al considerar el peso maduro y el SBW adecuados, las necesidades energéticas durante el crecimiento de borregos Pelibuey son similares a las de ovinos de razas lanares. Es posible que los programas de mejoramiento genético hayan incrementado el potencial productivo de esta raza, obteniéndose ganancias de peso similares a las razas lanares, lo que a su vez permite establecer criterios de comparación más objetivos, obteniendo como resultado necesidades energéticas similares.

El SRNS, subestima la predicción de la GDP en ovinos Pelibuey. Al ajustar el valor para el metabolismo basal en la ecuación que estima las necesidades de energía para el mantenimiento, disminuyéndolo a 0,052 , se corrige parcialmente la subestimación, y 


\section{DUARTE-VERA, SANDOVAL-CASTROYSARMIENTO-FRANCO}

al utilizar el valor de 0,039 se sobreestima la predicción; esto se representa con el valor de $u$ en la tabla IV. La $^{2} \mathrm{y} \mathrm{r}^{2}$ resistente tienen valores más elevados cuando se ajustaron los valores de a 1 , siendo mejores cuando se consideró el costo por eliminación del excedente de $\mathrm{N}$, indicando una mejor precisión para predecir la GDP; los valores de la $\mathrm{r}^{2}$ resistente y de la $\mathrm{r}^{2}$ no difieren mucho, indicando que son pocos los valores extremos de los datos analizados (tabla III).

La eficiencia del modelo (EM), mejoró considerablemente con el valor de 0,039 que incluye el costo por eliminación de $\mathrm{N}(0,42)$, como se presenta en la tabla IV, en donde el valor del modelo sin ajustar es cercano a cero.

El EEE estima la varianza del error aleatorio, siendo ligeramente menor en los modelos ajustados.
Los mejores valores para el CD, se obtuvieron con la disminución de a1, más el costo por eliminación de $\mathrm{N}$.

La MEP es una estimación confiable de la exactitud del modelo, por lo tanto en este caso, el menor valor indica que es mejor o más exacto, ocurriendo cuando se disminuye el valor de al y se considera dentro de éste el costo de energía para la eliminación del excedente de N. Al descomponer la MEP, observamos que la mayor desviación de la media de los datos predichos respecto a los observados, es con el modelo sin ajustar, tanto el valor de 0,052 como el de 0,039, reducen la desviación y por lo tanto mejoran la exactitud del modelo. El considerar el peso vivo vacío en el modelo, no mejora la predicción de la GDP en ninguno de los casos, solamente mejora parcialmente el

Tabla III. Resultado del análisis de regresión considerando los ajustes propuestos al modelo SRNS en machos Pelibuey. (Results of regression analysis considering model adjustments for Pelibuey males).

\begin{tabular}{llllll}
\hline & \multicolumn{1}{c}{ A } & \multicolumn{1}{c}{ B } & \multicolumn{1}{c}{$\mathrm{r}^{2}$} & $\mathrm{r}^{2}$ ajustada & EEE \\
\hline Modelo & & & & & \\
SRNS & $0,07154 \pm 0,01305$ & $0,81626 \pm 0,0952$ & 0,495 & 0,488 & 0,00264 \\
SRNS 0,052 c/u & $0,05748 \pm 0,01318$ & $0,76846 \pm 0,08055$ & 0,548 & 0,542 & 0,00236 \\
SRNS 0,052 s/u & $0,06225 \pm 0,0133$ & $0,68714 \pm 0,07555$ & 0,524 & 0,518 & 0,00249 \\
SRNS 0,052 PVV c/u & $0,07078 \pm 0,01259$ & $0,64886 \pm 0,0722$ & 0,518 & 0,512 & 0,00252 \\
SRNS 0,052 PVV s/u & $0,07484 \pm 0,01267$ & $0,58061 \pm 0,06744$ & 0,497 & 0,490 & 0,00263 \\
SRNS 0,039 c/u & $0,03950 \pm 0,01477$ & $0,71097 \pm 0,07380$ & 0,553 & 0,547 & 0,00234 \\
SRNS 0,039 s/u & $0,04554 \pm 0,01479$ & $0,63725 \pm 0,06912$ & 0,531 & 0,525 & 0,00245 \\
SRNS 0,039 PVV c/u & $0,05450 \pm 0,01387$ & $0,60451 \pm 0,06545$ & 0,532 & 0,526 & 0,00245 \\
SRNS 0,039 PVV s/u & $0,05973 \pm 0,01389$ & $0,54219 \pm 0,06127$ & 0,511 & 0,504 & 0,00256 \\
\hline
\end{tabular}

SRNS= Modelo Small Ruminant Nutrition System; SRNS 0,052 c/u= Modelo ajustando el factor a1 considerando el costo de energía para eliminar el excedente de N; SRNS 0,052 s/u= Modelo ajustando el factor a1, sin considerar el costo de energía para eliminar el excedente de N; SRNS 0,052 PVV c/u= Modelo ajustando el factor a1, el peso vivo vacío y considerando el costo de energía para eliminar el excedente de N; SRNS 0,052 PVV s/u= Modelo ajustando el factor a1 y el peso vivo vacío, sin considerar el costo de energía para eliminar el excedente de N; SRNS 0,039 $\mathrm{c} / \mathrm{u}=$ Modelo ajustando el factor a1 considerando el costo de energía para eliminar el excedente de N; SRNS 0,039 s/u= Modelo ajustando el factor a1, sin considerar el costo de energía para eliminar el excedente de N; SRNS 0,039 PVV c/u= Modelo ajustando el factor a1, el peso vivo vacío y considerando el costo de energía para eliminar el excedente de N; SRNS 0,039 PVV s/u= Modelo ajustando el factor a1 y el peso vivo vacío, sin considerar el costo de energía para eliminar el excedente de $\mathrm{N}$.

EEE= Error estándar de la estimación.

Archivos de zootecnia vol. 58, núm. 224, p. 678. 


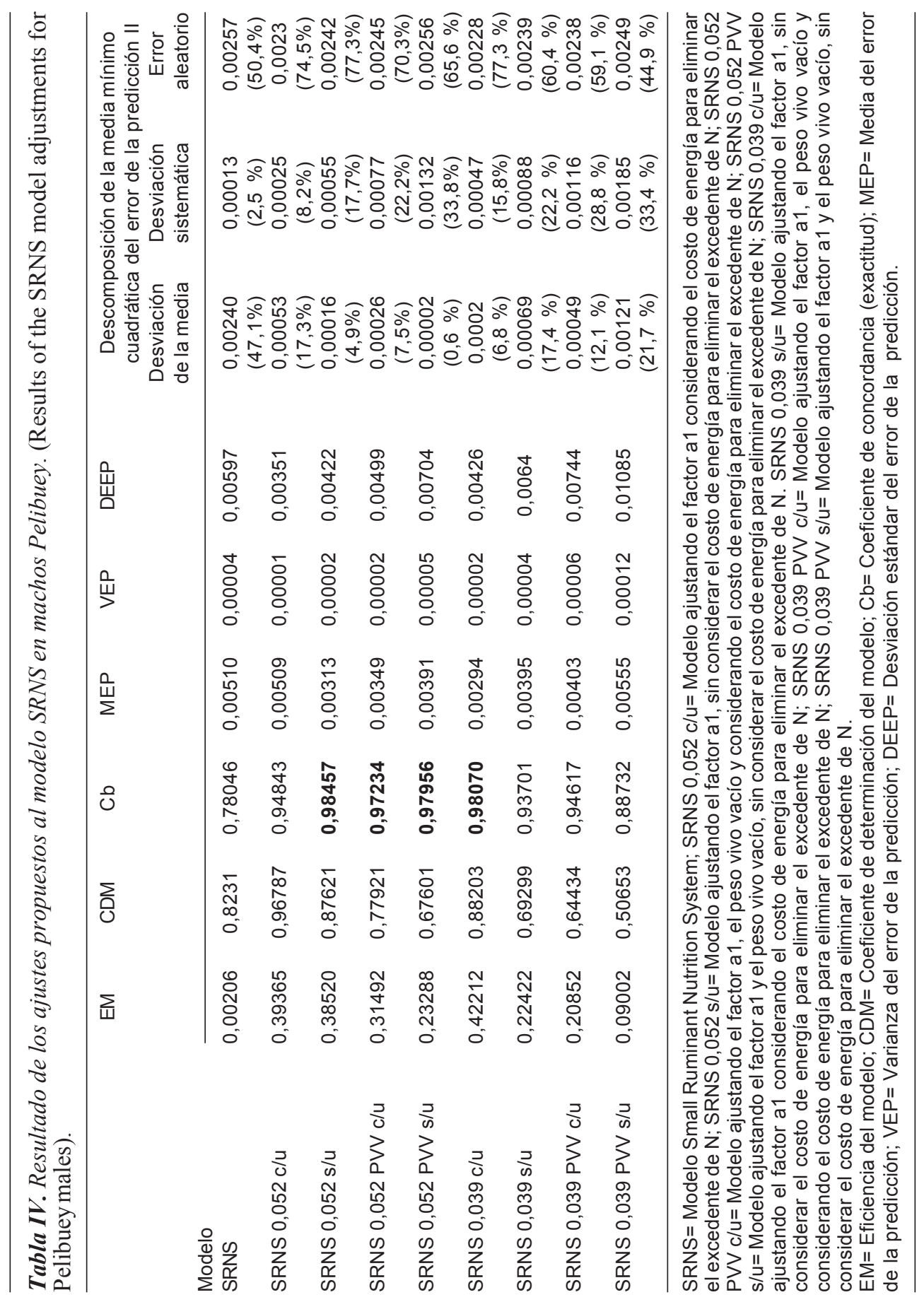




\section{DUARTE-VERA, SANDOVAL-CASTROYSARMIENTO-FRANCO}

coeficiente de concordancia $(\mathrm{Cb})$ cuando se emplea el valor de $\mathrm{a} 1=0,052$, indicando que mejora la precisión, como se puede observar en la tabla III.

\section{CONCLUSIONES}

Con base en los resultados anteriores se concluye que:

1. El valor del metabolismo basal en este trabajo (calculado con la información de la base de datos), es menor en ovinos de pelo (Pelibuey) que en razas lanares, al considerar el valor de a1=0,039 Mcal de EM/ $\mathrm{kg}^{0,75}$ (en la ecuación para estimar las nece-

\section{BIBLIOGRAFÍA}

Analla, M. 1980. Model validation through the linear regression fit to actual versus predicted values. Agr. Syst., 57: 115-119.

ARC. 1980. The nutrient requirements of ruminant livestock. $\mathrm{N}^{\circ} 2$ Ruminants. Technical Review Agricultural Research Council Working Party. Commonwealth Agricultural Bureaux. Farmham Royal. UK.

AFRC. 1993. Energy and protein requirements of ruminants. An advisory manual prepared by the AFRC Technical Committee on Responses to Nutrients. CAB International. Wallingford. UK.

Bores, Q.R., A.A.M. Martínez y R.A. Castellanos. 1988. Crecimiento compensatorio en el borrego Pelibuey. Tec. Pecu. Mex., 26: 8-15.

Bores, R.Q., M.A.Velázquez y M.A. Heredia. 2001. Evaluación de niveles energéticos y pesos de sacrificios de corderos terminales Suffolk $x \mathrm{~Pb}$. Segundo Congreso Latinoamericano de especialistas de pequeños rumiantes y camélidos sudamericanos. Memoria electrónica. Mérida Yuc.

Cannas, A., L.O. Tedeschi, D.G. Fox, A.N. Pell and P.J. Van Soest. 2004. A mechanistic model for predicting the nutrient requirements and feed biological values for sheep. J. Anim. Sci., 82: 49169

Cannas, A., L.O. Tedeschi, A.S. Atzori and D.G. Fox. 2006. Prediction of energy requirement for growing sheep with the Cornell net carbohydrate and protein system. In: Kebreab, E., Dijkstra, J., Bannink, A., Gerrits, W.J.J. and France, J. (Eds.). sidades de mantenimiento en el SRNS), mejoran la precisión y la exactitud de la estimación de la GDP en machos en crecimiento.

2. El ajuste por el contenido gastrointestinal no presenta ventajas para utilizarse en el modelo.

3. En general, la mejor respuesta se obtiene cuando se considera el gasto energético para eliminar el excedente de $\mathrm{N}$ en la ecuación.

4. Las adecuaciones propuestas permiten emplear el SRNS en ovinos de pelo Pelibuey en la etapa de crecimiento.

Nutrient digestion and utilization in farm animals Modelling approaches. CABI Publishing. Wallingford. UK. pp. 99-113

CONAGUA. 2005. Comisión Nacional del Agua, Gerencia Regional Península de Yucatán, México. Banco de datos, Mérida Yucatán, México. Abril de 2005.

Cohen, J.A. 1960. Coefficient of agreement for nominal scales. Educ. Physiol. Measur., 20: 3746.

CSIRO. 1990. Feeding standards for Australian livestock. CSIRO Publications. Melbourne. Australia.

Duarte, F., C.A. Sandoval-Castro and L. Sarmiento-Franco. 2007. Estimation of mature live weight of Mexican Pelibuey sheep. Proceedings of the British Society of Animal Science. Southport. UK. p. 135.

Duarte-Vera, F., C. Sandoval-Castro y L. Sarmiento-Franco.2008. Evaluación del modelo CNCPS$S$ para predecir el crecimiento del borrego Pelibuey. Rev. Cient. Fac. Cienc. Vet., 18: 296304.

Freer, M.M. and J.R. Donnelly. 1997. Grazplan: Decision support system for Australian grazing enterprises: II. The animal biology model for feed intake, production and reproduction and the graze. Fedd DSS. Agr. Syst. 54: 77-126.

Harrison, S.R. 1990. Regression of a model on real-system output: an invalid test of model validity. Agr. Syst., 34: 183-190.

INRA. 1978. Alimentation des ruminants. INRA 


\section{MODELO PARA LA PREDICCIÓN DE LA GANANCIA DE PESO EN OVINOS PELIBUEY}

Publications. Versailles. France.

Martínez, A.A.A., Q.R. Bores, R.A. Castellanos 1987. Zoometría y predicción corporal de la borrega Pelibuey. Tec. Pecu. Mex. 25: 72-84.

Martinez, A.A.M., T.J. Soriano e A.S. Shimada. 1985. Crecimiento de borregos Pelibuey alimentados con rastrojo de maíz tratado con amoniaco anhidro. Tec. Pecu. Mex., 48: 54-61.

Mayer, D.G. and D.G. Butler. 1993. Statistical validation. Ecol. Model., 68: 21-32.

Mayer, D.G., M.A. Stuart and A.J. Swain. 1994. Regression of real-world data on model output: an appropriate overall test of validity. Agr. Syst. 45: 93-104.

Model Evaluation System. 2006. Versión 2.0.11.Texas A\&M University, Department of Animal Science. 230 Kleberg Center. TAMU. College Station. TX 77840 .

NRC. 1985. Nutrient requirements of sheep. Sixth revised edition. National academy press. Washington D.C. USA.

NRC. 1996. Nutrient requirement of beef cattle. Subcommittee on cattle nutrition, committee on animal nutrition board on agriculture National research council, $7^{\text {th }}$ rev. ed. National Academy of Sciences. National academy press. Washington, DC. USA.

Olthoff, J.C., G.E. Dickerson and J.A. Nienaber. 1989. Energy utilization in mature ewes from seven breeds with diverse production potential. J. Anim. Sci., 67: 2550-2564.

Partida Becerra, E. 1983. Evaluación del crecimiento compensatorio de borregos en etapa de finalización, mediante el uso de ensilaje de maíz amoniado. Tesis de licenciatura Mvs. UNAM.

Romano, M.J.L., G.J. Hernández y R.A. Castellanos. 1983. Repercusión del valor nutritivo de la dieta sobre el crecimiento del borrego Pelibuey. Tec. Pecu. Mex., 45: 67-79.

SAS. 1988. Statistical Analysis System. User's Guide (Release 6.03). SAS Inst. Inc. Cary NC, USA.

Solís, R.G.E., A.F. Castellanos, A.M. Velásquez and G.F. Rodríguez. 1991. Determination of nutricional requirements of growing hair sheep. Small Ruminant Res., 4: 115-125.

Tedeschi, L.O., D.G. Fox, A.N. Pell, L.D.P. Duarte and C. Boin. 2002. Development and evaluation of a tropical feed library for the Cornell Net Carbohydrate and Protein System Model. Sci. Agric., 59: 1-18.

Tedeschi, L.O. 2006. Assessment of the adequacy of mathematical models. Agr. Syst., 89: 225247.

Tedeschi, L.O. 2007. Mathematical nutrition models. Small Ruminant Nutrition System. http:// nutritionmodels.tamu.edu/srns.htm. Agosto de 2007. 